\title{
The Influence of Quantitative Parameters Measured on CT with Different Slice Thicknesses on Evaluating the Growth of Pulmonary Nodules
}

\author{
Jannatul Maoya Bashanti ${ }^{1, ~}$, Binjie Fu ${ }^{1}$, Wang Jia Li ${ }^{1}$, Mohammad Arman Hossain ${ }^{2}$, Fajin Lv ${ }^{1}$ \\ ${ }^{1}$ Department of Imaging and Nuclear Medicine, The First Affiliated Hospital of Chongqing Medical University, Chongqing, China \\ ${ }^{2}$ Department of Urology, The First Affiliated Hospital of Chongqing Medical University, Chongqing, China
}

Email address:

jannatalin@yahoo.com (J. M. Bashanti)

${ }^{*}$ Corresponding author

\section{To cite this article:}

Jannatul Maoya Bashanti, Binjie Fu, Wang Jia Li, Mohammad Arman Hossain, Fajin Lv. The Influence of Quantitative Parameters Measured on CT with Different Slice Thicknesses on Evaluating the Growth of Pulmonary Nodules. International Journal of Medical Imaging.

Vol. 9, No. 2, 2021, pp. 109-116. doi: 10.11648/j.ijmi.20210902.13

Received: April 30, 2021; Accepted: May 18, 2021; Published: May 26, 2021

\begin{abstract}
We did this study to investigate the effect of thick $(5 \mathrm{~mm})$ and thin $(1$ or $0.625 \mathrm{~mm})$ slice thickness of CT images on evaluating pulmonary nodules' growth to improve their diagnostic accuracy. The clinical and CT data of 251 patients with lung nodules and two follow-up CTs from October 2016 to October 2019 were analyzed retrospectively. Malignant nodules were confirmed by pathology, and benign nodules were confirmed by pathology or follow-up. Two radiologists double-blindly assessed the CT features (density, shape, lobes, border), maximum diameter, and volume of nodules on the thick (5MM) and thin $(\leq 1 \mathrm{MM})$ images of two follow-up CTs. We use One-way analysis of variance for quantitative data; the X2 test or FISHER exact probability method was used for qualitative data; and the ROC curve was used to analyze the diagnostic power of nodule size, volume, and change in differentiating benign and malignant lesions. Among 251 pulmonary nodules, 117 (46.6\%) benign nodules and $134(53.3 \%)$ malignant nodules. During the CT follow-up, the volume measured on the thick-section image, the diameter, and the volume measured on the thin-section image were statistically different in benign and malignant lung nodules $(\mathrm{P}<0.001)$. In contrast, the diameter measured on the thick-section image was similar between these two groups $(\mathrm{P}=0.328)$. For benign and malignant pulmonary nodules, the diameter, volume, and change measured on the thin-section image were significantly larger than the thick-section image's data $(\mathrm{P}<0.001)$. The ROC curve showed that the diagnostic efficiency of volume was higher compared to the diameter. There were significant differences in nodule type, density change, shape, lobulation, and pleural retraction between benign and malignant nodules for CT features. Accurately assessing the volume changes combined with CT characteristics will help improve lung nodules' diagnosis accuracy. Volume measured on thin-section $(1 \mathrm{~mm})$ CT images is the best quantitative parameter for assessing the change of pulmonary nodules. Combining Volume change with CT characteristics would help to improve the diagnostic accuracy.
\end{abstract}

Keywords: Computed Tomography, Volume Measurements, Diameter Measurements, Malignant Tumors, Benign Nodules, MDCT

\section{Introduction}

The pulmonary nodule is a spherical, well-circumscribed, radiographic opacity measuring within $3 \mathrm{~cm}$ in diameter and is surrounded by aerated lung. Associated atelectasis, hilar enlargement, or pleural effusion is not present.[1] Among malignant tumors, lung cancer gets the highest ranking, and it is the leading cause of mortality. Therefore, early diagnosis of lung cancer is essential.[2] Studies have shown that in patients with malignant tumors outside the lungs, the proportion of lung nodules detected by thin-layer CT can reach $60-75 \%$.[4] If a pulmonary nodule is detected, especially from metastasis, the possibility of being considered malignant is significantly increased, which leads to an increased risk of misdiagnosis to a great extent. Detecting small pulmonary nodules highly suggestive of lung cancer is increased due to widespread 
computed tomography (CT). There is a massive preference for MDCT (multi-slice spiral CT). MDCT increases the number of images reducing the layer thickness and improving image quality.[3] The National Lung Screening Trial has reported that patients with screening low-dose CT (LDCT) scanning showed reducing relative risk of death from lung cancer by $20 \%$.[5]

Patients with malignant tumors or newly grown pulmonary nodules are of great significance in tumor staging, treatment choice, and prognostic evaluation. Therefore, summarizing the $\mathrm{CT}$ characteristic manifestations of pulmonary nodules in patients with malignant tumors and improving their diagnostic and differential diagnosis capabilities is of high clinical value.

\section{Methods and Materials}

\subsection{Research Object}

A retrospective analysis of patients' chest CT data with diagnosed pulmonary nodules in First Affiliated Hospital of Chongqing Medical University from October 2016 to October 2019 was done. Inclusion criteria: (1) Thin CT and thick CT data were only considered. (2) Lung nodule $<3 \mathrm{~cm}$. (3) Benign lung nodules from tuberculosis, pneumonia, pneumoconiosis, sarcoidosis was taken. (4) Malignant nodules, primary lung cancer, and metastasis causing lung nodules were taken. (5) Lung nodules with precise pathological diagnosis. Exclusion criteria: (1) Diffuse lung disease (2) Artifacts were affecting the actual image analysis (3) Partly solid nodules with solid content more than $3 \mathrm{~mm}$. Finally, 251 lung nodules were enrolled in this study.

\subsection{CT Examination}

A Siemens 128-MDCT scanner (Somatom Definition Flash, siemens healthcare) was used for chest CT examination. Patients were supine with both upper limbs raised. The scan was ranged from the thorax entrance to the lung base level at the tip of the posterior costophrenic angle. The scan is completed at the end of deep inhalation. The scanning parameters are as follows: Tube voltage 110-120 kvp, using care does 4D automatic tube current technology, pitch: 1.3, rack speed $0.6 \mathrm{~s} / \mathrm{r}$, Acquisition matrix: 512 x 512, reconstructed with $1 \mathrm{~mm}$ or less than $1 \mathrm{~mm}$ slice thickness.

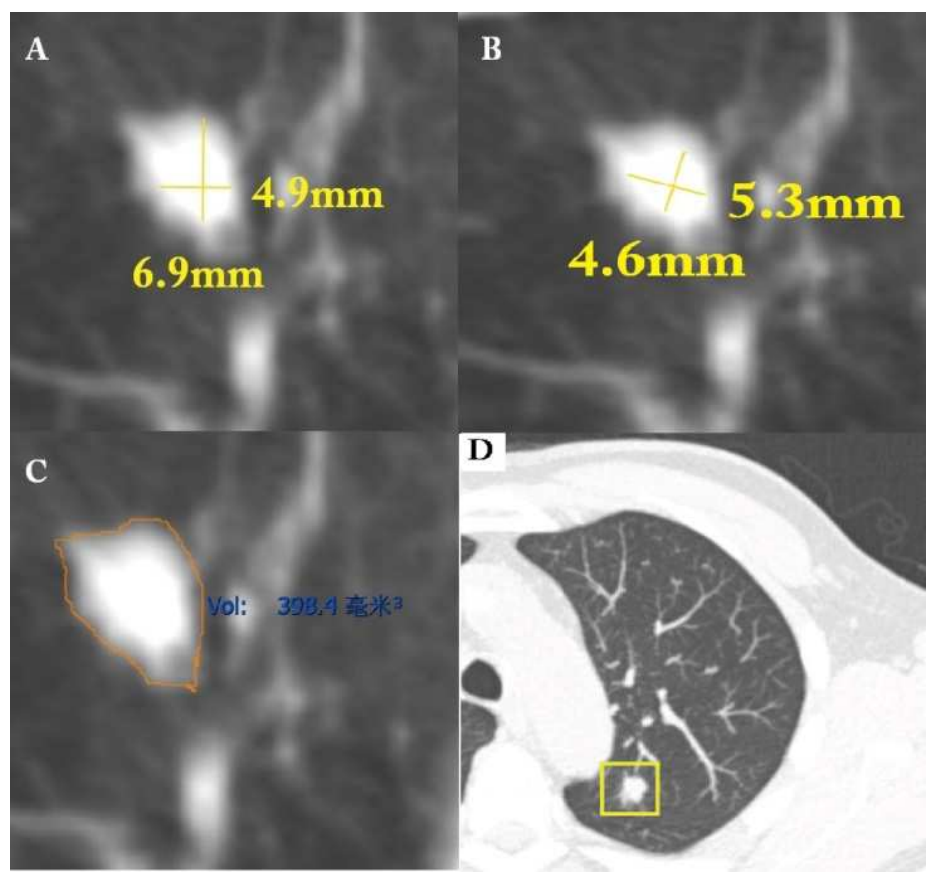

Figure 1. Transverse images of a solid pulmonary nodule and its location. Two possible diameter measurements (A and B) and manually assessed volume (C). The multiplanar evaluation of nodule size is essential to document asymmetrical growth of nodules. All measurements were taken in $1 \mathrm{~mm}$ slice thickness.

\subsection{Image Analysis}

Two experienced chest radiologists individually evaluated CT images of all patients. The nodules' final pathological results and the patient's relevant medical history were unknown at reading. When the opinions differed, a unified opinion was reached after discussion. The assessment includes diameter, volume, nodule position, density (solidity, sub solidity), shape (round or oval, irregular), density uniformity (even, mixed), boundary (clear, blurry), lobulation (present or not), spiculation (present or not), the relationship with the pleura (clinging with pleura or without clinging).

\subsection{CT Technique for Volume and Diameter Measurement}

For volume measurement, the Region of interest (ROI) was first defined by outlining the nodule borders section by section, and manually the volume was measured. The diameter was measured by taking an average of long and short axis measurements. A single radiologist performed volume measurement and segmentation assessment, and then two senior radiologists who are experts as in pulmonary radiologist measured it 2 to 3 times, came to a unified decision. CT technique for diagnosing and accurately 
evaluating pulmonary nodules requires contiguous thin section evaluation, optimally with 1-mm sections. A detected nodule's subsolid or solid nature can be confirmed and discriminated against with thin sections as small solid nodules appear faint due to partial volume effect on thicker sections. Thin sections enable a more accurate assessment of nodule size and the presence and size of any solid component factors correlating with patient prognosis and aiding in determining management.[6] Remarkably, it has been suggested that thin-section images increase sensitivity in detecting subsolid nodules, specifically pure ground-glass nodules, and avoid the misinterpretation of solid nodules as subsolid nodules (SSNs) [6, 7].

\subsection{Statistical Methods}

SPSS 20.0 software was used for statistical analysis. Measurement of data is expressed by means \pm standard deviation or interquartile range (IQR) and analyzed by Wilcoxon test; count data are expressed by rate (\%) and analyzed with Pearson chi-square or Fischer exact test. To compare the differences among benign and malignant nodules with diameter and volume and other morphological appearances of nodule $\mathrm{p}<0.05$ is indicated that statistically significant.

\section{Results}

\subsection{Clinical Characteristics of Patients}

Among 251 patients, 86 (34.3\%) were male including 43 (36.8\%) benign and 43 (32.1\%) malignant, and $165(65.7 \%)$ were female including $74(63.2 \%)$ benign and $91(67.9 \%)$ malignant. The average age of patients was $54.1 \pm 11.4$ years old (range: $30 \sim 79$ years old). Comparisons between study subjects with benign and malignant nodules for age and sex show no significance. Medical history of patients with malignant tumors: lung cancer, breast cancer, thyroid cancer, colorectal cancer, cervical cancer, endometrial cancer. Benign lesions were mainly tuberculosis, pneumonia, pneumoconiosis.

\subsection{Analysis of Diameter and Volume}

During the CT follow-up, the volume measured on the thick section $(5 \mathrm{~mm})$ image was statistically different in benign and malignant nodules $(\mathrm{p}<0.001)$, while the diameter measured on the thick section $(5 \mathrm{~mm})$ image was similar between these two groups $(\mathrm{P}=0.328)$. For benign and malignant pulmonary nodules, the diameter, volume, and their changes measured on the thin section $(\leq 1 \mathrm{~mm})$ image were significantly larger than the data measured on the thick section $(5 \mathrm{~mm})$ image $(\mathrm{p}<0.001)$.

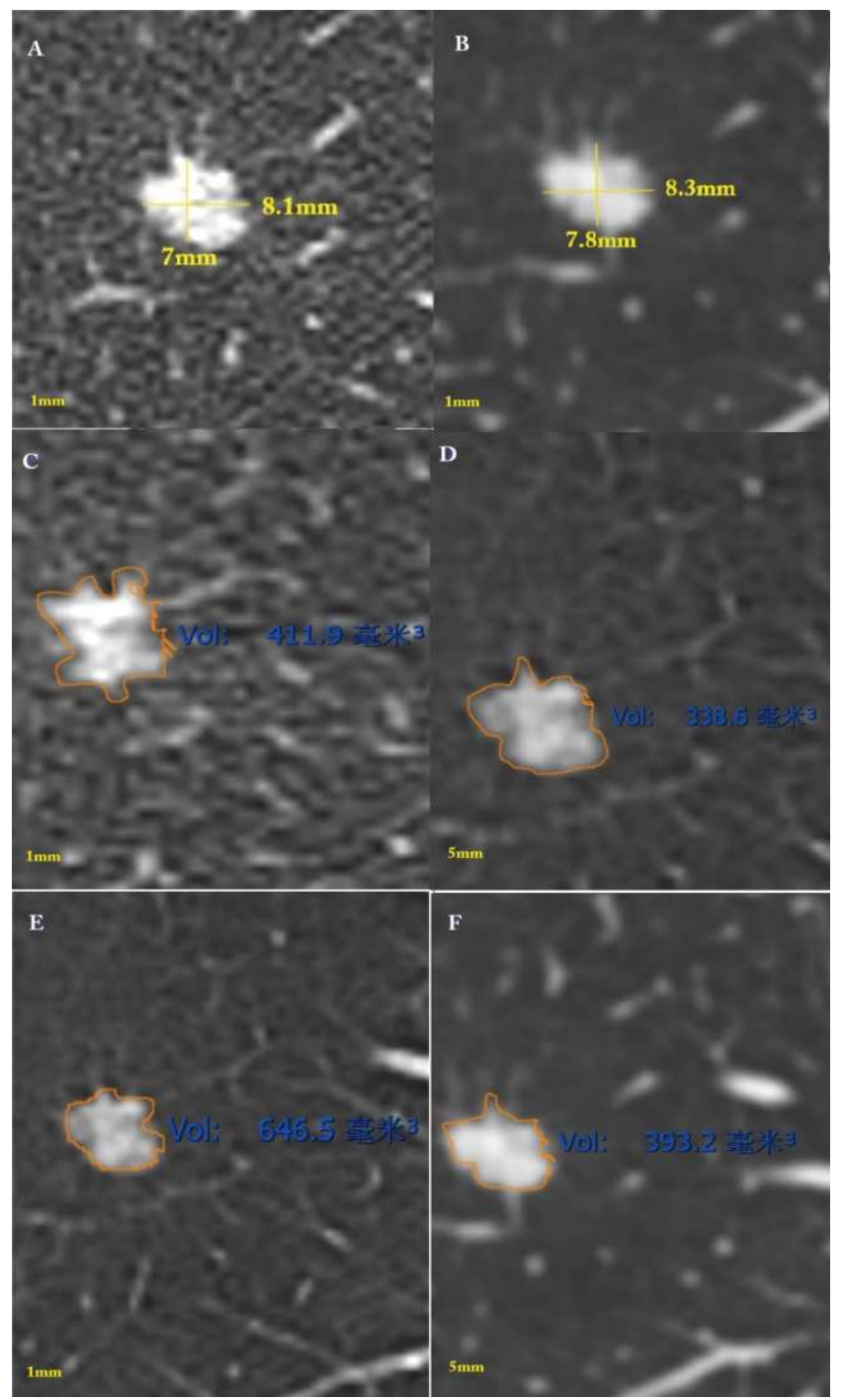

Figure 2. Axial diameter of an asymmetrical small solid nodule located apical posterior segment of right middle lobe in a 37-year-old female patient. Measurements were taken in 3 months intervals ( $A$ and B), showing a significantly less difference. Initial $(C$ and $D)$ volume and follow-up volume ( $E$ and $F$ ) measured in $1 \mathrm{~mm}$ and $5 \mathrm{~mm}$ slice thickness.

Table 1. Volume and Diameter changes with a different slice thickness.

\begin{tabular}{lll}
\hline Screening method & Patient with benign nodule $(\mathbf{n}=117)$ & Patient with malignant nodule $(\mathbf{n}=134)$ \\
\hline Dchange_5MM & $1.33 \pm 1.64$ & $1.49 \pm 1.13$ \\
Dchange_1MM & $0.89 \pm 1$ & $1.06 \pm 0.91$ \\
Vchange_5MM & $97.75 \pm 121.1$ & $230.72 \pm 327.51$ \\
Vchange_1MM & $174.55 \pm 212.97$ & $323.83 \pm 392.22$ \\
\hline
\end{tabular}

Note. Data are presented as n (\%) or Means \pm SD.

It was calculated with Wilcoxon rank-sum test.

D change $(5 \mathrm{~mm})=$ diameter change in $5 \mathrm{~mm}$ slice thickness.

$D$ change $(1 \mathrm{~mm})=$ diameter change in $1 \mathrm{~mm}$ slice thickness

$V$ change $(5 \mathrm{~mm})=$ volume change in $5 \mathrm{~mm}$ slice thickness,

$\mathrm{V}$ change $(1 \mathrm{~mm})=$ volume change in $1 \mathrm{~mm}$ slice thickness. 
Table 2. Comparisons of benign nodules with a different slice thickness.

\begin{tabular}{lll}
\hline Benign nodules (n=117) & $\mathbf{5 ~ M M ~}$ & 1 MM \\
\hline D1 & $7.37 \pm 3.07$ & $8.4 \pm 3.45$ \\
Median (P25, P75) & $7(5.1,8.85)$ & $8.05(6.25,10.25)$ \\
D2 & $7.97 \pm 3.43$ & $8.84 \pm 3.42$ \\
Median (P25, P75) & $7.55(5.5,10.15)$ & $8.4(6.7,10.85)$ \\
D change & $1.33 \pm 1.64$ & $0.89 \pm 1$ \\
Median (P25, P75) & $1.1(0.6,1.9)$ & $0.55(0.2,1.2)$ \\
V1 & $475.97 \pm 654.05$ & $563.86 \pm 720.75$ \\
Median (P25, P75) & $239.7(169.3,447.7)$ & $291.5(213.5,618.3)$ \\
V2 & $522.62 \pm 700.81$ & $663.82 \pm 891.88$ \\
Median (P25, P75) & $277.8(166.7,500.5)$ & $345.4(202.3,702.6)$ \\
V change & $97.75 \pm 121.1$ & $174.55 \pm 212.97$ \\
Median (P25, P75) & $59.2(28.2,113.8)$ & $101(45,198.8)$ \\
\hline
\end{tabular}

Note. Data are presented as $n(\%)$ or Means \pm SD.

Calculated with Wilcoxon rank-sum test

D change $(5 \mathrm{~mm})=$ diameter change in $5 \mathrm{~mm}$ slice thickness.

$D$ change $(1 \mathrm{~mm})=$ diameter change in $1 \mathrm{~mm}$ slice thickness

$V$ change $(5 \mathrm{~mm})=$ volume change in $5 \mathrm{~mm}$ slice thickness,

$\mathrm{V}$ change $(1 \mathrm{~mm})=$ volume change in $1 \mathrm{~mm}$ slice thickness.

Table 3. Comparisons of malignant nodules with a different slice thickness.

\begin{tabular}{llll}
\hline Malignant nodule (n=134) & $\mathbf{5 M M}$ & 1MM & P-value \\
\hline D1 & $9.17 \pm 3.85$ & $9.95 \pm 3.91$ \\
Median (P25, P75) & $8.5(6.8,10.7)$ & $9.28(7.55,11.8)$ \\
D2 & $10.65 \pm 4.29$ & $11.01 \pm 4.23$ & \\
Median (P25, P75) & $9.53(7.9,12.8)$ & $10.13(8.45,12.75)$ & 0.001 \\
D change & $1.49 \pm 1.13$ & $1.06 \pm 0.91$ & $0.8(0.35,1.5)$ \\
Median (P25, P75) & $1.2(0.75,1.85)$ & $1112.44 \pm 1382.03$ \\
V1 & $976.15 \pm 1305.65$ & $596.45(349.7,1191)$ \\
Median (P25, P75) & $489.2(292,1057.5)$ & $1436.27 \pm 1654.33$ \\
V2 & $1206.86 \pm 1511.38$ & $864.75(512.7,1486.4)$ \\
Median (P25, P75) & $644.3(425.2,1254.3)$ & $323.83 \pm 392.22$ \\
V change & $230.72 \pm 327.51$ & $187.4(104.3,399)$ \\
Median $(P 25$, P75) & $114.15(59.9,298.7)$ & $<0.001$ \\
\hline
\end{tabular}

Note. Data are presented as $\mathrm{n}(\%)$ or Means \pm SD.

Calculated with Wilcoxon rank-sum test

D change $(5 \mathrm{~mm})=$ diameter change in $5 \mathrm{~mm}$ slice thickness.

$D$ change $(1 \mathrm{~mm})=$ diameter change in $1 \mathrm{~mm}$ slice thickness

$V$ change $(5 \mathrm{~mm})=$ volume change in $5 \mathrm{~mm}$ slice thickness,

$V$ change $(1 \mathrm{~mm})=$ volume change in $1 \mathrm{~mm}$ slice thickness.

The volume and diameter changes with a different slice thickness were shown in Table 1. The diameter change of thin $(\leq 1 \mathrm{~mm})$ and thick $(5 \mathrm{~mm})$ section thickness were $0.89 \pm 1 \mathrm{~mm}$, $1.06 \pm 0.91 \mathrm{~mm}$ and $1.06 \pm 0.91 \mathrm{~mm}, 1.49 \pm 1.13 \mathrm{~mm}$. The volume change of thin $(\leq 1 \mathrm{~mm})$ and thick $(5 \mathrm{~mm})$ section thickness for benign and malignant nodules were $174.55 \pm 212.97 \mathrm{~mm}^{3}$, $97.75 \pm 121.1 \mathrm{~mm}^{3}$; and $323.83 \pm 392.22 \mathrm{~mm}^{3}, 230.72 \pm 327.51$ $\mathrm{mm}^{3}$. The volume change measured on the thick and thin-section image and the diameter change were statistically different in benign and malignant lung nodules $(\mathrm{P}<0.05)$.

Volume change in $5 \mathrm{~mm}$ slice thickness area under the curve (AUC) was 0.692 with $p$-value $<0.001$ with sensitivity and specificity $(95 \% \mathrm{CI} ; 83.6 \%$ and $76 \%)$. For $1 \mathrm{~mm}$, slice thickness AUC was 0.672 , and sensitivity and specificity were $(95 \% \mathrm{CI}$; $76.1 \%$ and $50.4 \% \mathrm{P}<0.001$ ), which differed significantly. Diameter change in $1 \mathrm{~mm}$ and $5 \mathrm{~mm}$ slice thickness AUC were 0.532 and 0.581 , and sensitivity and specificity were $63.4 \%$ and $36.56 \%$; $\mathrm{p}$ value $=0.027$ for $1 \mathrm{~mm}$ slice thickness and $68.7 \%$ and $42.7 \%$; p-value $>0.05$ for $5 \mathrm{~mm}$ slice thickness respectively which is below the reference line. This study found that volume gives a more precise and accurate value than manual diameter measurement. The growth of nodules can be predicted more accurately by adding this parameter in clinical practice.

Sensitivity and specificity for diameter and volume change are shown in table 4, and AUC (area under the curve) is shown in Table 5.

Table 4. Sensitivity and specificity for diameter and volume change.

\begin{tabular}{lll}
\hline VARIABLE & SENSITIVITY \% & SPECIFICITY \% \\
\hline D change $(5 \mathrm{~mm})$ & 68.7 & 42.7 \\
D change $(1 \mathrm{~mm})$ & 63.4 & 36.56 \\
V change $(5 \mathrm{~mm})$ & 83.6 & 47 \\
V change $(1 \mathrm{~mm})$ & 76.1 & 50.4 \\
\hline
\end{tabular}

Table 5. Area Under the Curve.

\begin{tabular}{lc}
\hline Test Result Variable (s) & Area \\
\hline D change $5 \mathrm{~mm}$ & .532 \\
D change $1 \mathrm{~mm}$ & .581 \\
Vol change $(5 \mathrm{~mm})$ & .692 \\
Vol change $(1 \mathrm{~mm})$ & .672 \\
\hline
\end{tabular}




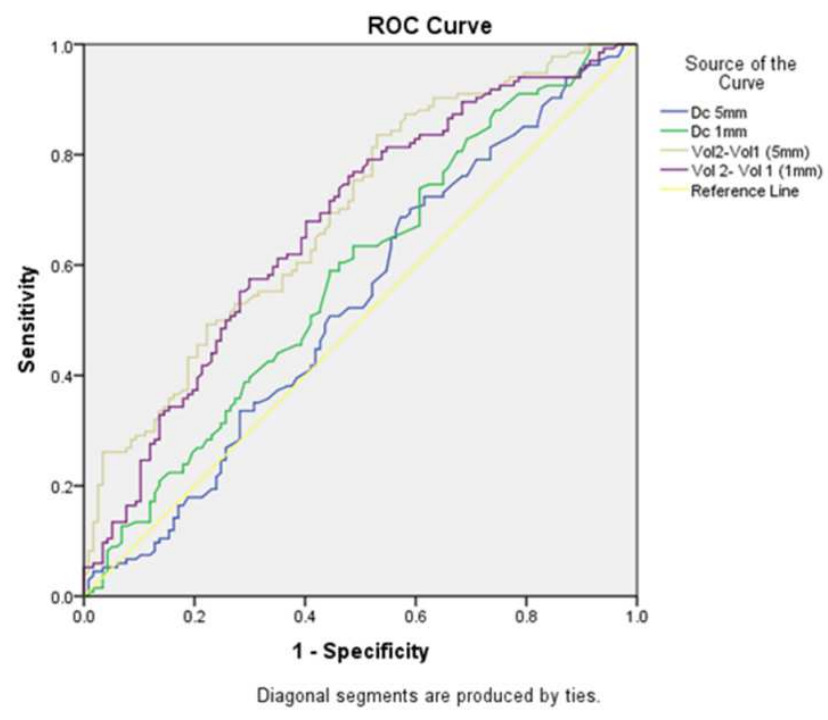

Figure 3. The ROC curve showed that the diagnostic efficiency of volume was higher compared to the diameter. There were significant differences in nodule type, density change, shape, lobulation, and pleural retraction between benign and malignant nodules for CT features.

\subsection{CT characteristics of Pulmonary Nodules with Different Pathology}

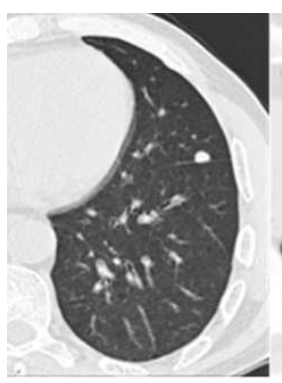

A

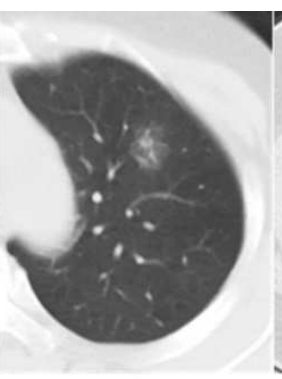

B

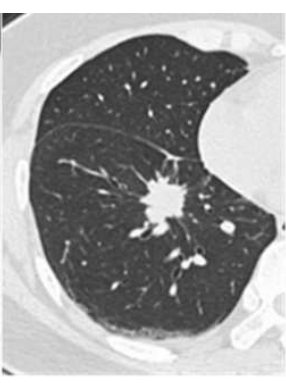

Figure 4. (A) Patient, 61 years old, after rectal cancer surgery, the left upper lobe with round solid nodules with uniform density, smooth edges, clear boundaries, pathologically confirmed as metastases. (B) A 63-year-old female patient, after breast cancer surgery, had a subsolid nodule in the upper lobe of the left lung, with mixed density and transparent borders, pathologically confirmed as primary lung cancer. (C) Patient female, 62 years old, after surgery of nasopharyngeal carcinoma, the right lower lobe of the right lung nodule, uniform density, clear border, rough edges, pathologically confirmed to be Primary lung cancer.

The distribution of nodule location and CT appearances of pulmonary nodules of different pathological types are shown in Table. Among 251 analyzed nodules, 134 (53.3\%) malignant nodules were confirmed by pathological examination (Hematoxylin-eosin and immunohistochemistry), $117(46.6 \%)$ benign nodules in which cases of tuberculosis, pneumonia, pneumoconiosis, benign tumors like hamartomas, pseudotumor involved. Out of 117 benign nodules, 63 (53.8\%) were solid, and 54 (46.2\%) were subsolid nodules. 49 (41.95\%) were irregular, and $68(58.1 \%)$ were round or oval. On benign nodules, spiculations presented in only $4(3.4 \%)$ nodules, and only $3(2.6 \%)$ nodules were lobulated. $5(4.3 \%)$ nodules were found with mixed density with $37(31.6 \%)$ blurry margins. On the other hand, only $36(26.9 \%)$ and 98 (73.1\%) were solid and subsolid found in malignant nodules, respectively, in which 81 $(60.4 \%)$ were irregular, and $53(39.6 \%)$ nodules were in round or oval shape. Nodules edges were spiculated 18 (13.4\%), and $8(6 \%)$ were lobulated, $31(23.1 \%)$ with blurry margins, and $103(76.9 \%)$ malignant nodules were clear margins. After pairwise comparisons nodules type, nodule forms spiculated nodules both benign and malignant nodules were statistically significant $(\mathrm{P}=<0.05)$. We found no significant difference in the distribution of different types of nodules in each lung lobe and its positional relationship with the pleura $(\mathrm{p}>0.05)$. The pleural retraction was present only in $6(5.1 \%)$ in benign and $21(15.7 \%)$ in malignant nodules, which is statistically significant. Therefore, the presence of air bronchogram in benign nodules $22(8.8 \%)$ and malignant $16(6.4 \%)$ nodules were not statistically significant $(\mathrm{p}<0.05)$. CT features of different pulmonary nodules are given in table 6 .

Table 6. CT findings of internal features for different pulmonary nodules.

\begin{tabular}{|c|c|c|c|}
\hline CT features & $\begin{array}{l}\text { Benign nodule } \\
(\mathrm{n}=117)\end{array}$ & $\begin{array}{l}\text { Malignant } \\
\text { nodule }(n=134)\end{array}$ & P-value \\
\hline Age (y) & $53.2 \pm 11.7$ & $54.8 \pm 11.1$ & 0.262 \\
\hline \multicolumn{4}{|l|}{ Gender } \\
\hline Male & $43(36.8)$ & $43(32.1)$ & 0.438 \\
\hline Female & $74(63.2)$ & $91(67.9)$ & \\
\hline \multicolumn{4}{|l|}{ Nodule Type } \\
\hline Solid & $63(53.8)$ & $36(26.9)$ & $<0.001$ \\
\hline Subsolid & $54(46.2)$ & $98(73.1)$ & \\
\hline \multicolumn{4}{|l|}{ Shape } \\
\hline Irregular & $49(41.9)$ & $81(60.4)$ & 0.003 \\
\hline Round or oval & $68(58.1)$ & $53(39.6)$ & \\
\hline \multicolumn{4}{|l|}{ Spiculation } \\
\hline Yes & $4(3.4)$ & $18(13.4)$ & 0.005 \\
\hline No & $113(96.6)$ & $116(86.6)$ & \\
\hline \multicolumn{4}{|l|}{ Lobulation } \\
\hline Yes & $3(2.6)$ & $8(6.0)$ & 0.189 \\
\hline No & $114(97.4)$ & $126(94.0)$ & \\
\hline \multicolumn{4}{|l|}{ Location } \\
\hline RUL & $46(39.3)$ & $53(39.6)$ & \\
\hline RML & $16(13.7)$ & $10(7.5)$ & \\
\hline RLL & $15(12.8)$ & $17(12.7)$ & 0.535 \\
\hline LUL & $28(23.9)$ & $40(29.9)$ & \\
\hline LLL & $12(10.3)$ & $14(10.4)$ & \\
\hline \multicolumn{4}{|l|}{ Pleural retraction } \\
\hline Yes & $6(5.1)$ & $21(15.7)$ & 0.007 \\
\hline No & $111(94.9)$ & $113(84.3)$ & \\
\hline \multicolumn{4}{|l|}{ RELATION TO PLEURA } \\
\hline Clinging with pleura & $25(21.4)$ & $27(20.1)$ & 0.812 \\
\hline Far away from pleura & $92(78.6)$ & $107(79.9)$ & \\
\hline \multicolumn{4}{|l|}{ Density uniformity } \\
\hline Even & $111(95.7)$ & $124(92.5)$ & 0.295 \\
\hline Mixed & $5(4.3)$ & $10(7.5)$ & \\
\hline \multicolumn{4}{|l|}{ Air bronchogram } \\
\hline Yes & $22(8.8)$ & $16(6.4)$ & 0.130 \\
\hline No & $95(37.8)$ & $118(47.0)$ & \\
\hline \multicolumn{4}{|l|}{ Boundary } \\
\hline Blurry & 37 (31.6) & $31(23.1)$ & 0.131 \\
\hline Clear & $80(68.4)$ & $103(76.9)$ & \\
\hline
\end{tabular}

1. T-test, Pearson's chi-square test done. $\mathrm{RUL}=$ right upper lobe, $\mathrm{RML}=$ right middle lobe, $\mathrm{RLL}=$ right lower lobe, $\mathrm{LUL}=$ left upper lobe, $\mathrm{LLL}=$ left lower lobe,

2. the data in the Table are all expressed as n (\%) 


\section{Discussion}

For volumetric analysis to be helpful, it must be both accurate and reproducible.[8] Analysis of lung nodules shows that using lung nodule volume as a parameter to predict nodule growth is an important index compared with the diameter. Although manual volume measurement slice per slice is time-consuming for diagnosis accuracy, it is a good predictor for nodule growth. Volume measurement gives accurate value if the lesion area is appropriately adjusted. Malignant nodules invasiveness can be predicted, and benign nodules, which decrease in volume, can be treated with follow-up series while malignant lesions need urgent surgery. Therefore, it is essential to diagnose accurate nodular growth [13]. This study focuses on nodule growth depending on both diameter and volume. Volume and diameter initial and follow-up measurements were done with changes in them to assess the accuracy. However, diameter did not show changes in indeterminate nodules that much, significantly less even in malignant nodules, especially malignant nodules in $5 \mathrm{~mm}$ slice thickness; a significant change was noted in volume [18]. Therefore, it can be said that adding volume as a growth index would not be wrong. Mehta et al. reported that using volumetry-based nodule volume significantly increased a validated lung cancer risk prediction model's predictive ability compared with using diameter measurements [19]. The addition of volume with other parameters to learn about lung nodules' growth has a significant impact. This study was done in the Swensen model comparing with other models [16]. Revenel et al. reported that volume less than or equal to $1 \mathrm{~mm}$ slice thickness shows maximum precision to detect nodule size, significantly smaller nodules [20]. They also reported very little Intra and inter-observer variability using less than $1 \mathrm{~mm}$ slice thickness; thus, it represents close to the nodule's actual size. Estimating nodule size by volume measurements is a presumptive method compared to manual diameter measurements [17]. Since there is a limited number of studies that compare inter-and intrareader variability of small-sized lung nodule measurements, it is relatively easy to ensure the standardization of imaging parameters in controlled experiments [21]. However, in clinical practice, these parameters may vary. Besides, the time necessary to reconstruct data sets in different ways may hinder using volumetric analysis. Thus, it is necessary to understand the appropriate reconstruction parameters that should be used on measurement accuracy [22]. However, section thickness has a significant effect on bias and precision, particularly for smaller nodules. In this study, the measurements obtained using the $0.625-\mathrm{mm}$ section thickness were superior, with minimal bias and maximal precision across all reference-standard diameters [23]. Use of the $5.00-\mathrm{mm}$ section thickness yielded inferior measurements for 5.00-mm and smaller nodules [24]. Thus, for unbiased and precise volume estimation of small $(<5 \mathrm{~mm})$ pulmonary nodules, a section thickness of no greater than $\leq 1.00 \mathrm{~mm}$ should be used. The use of a $5.00-\mathrm{mm}$ section thickness is discouraged.[8] Han et al. reported in phantom studies volume measurements give more accurate results than manual diameter measurements, including thin slice thickness, usually less than $1 \mathrm{~mm}$ slice thickness [25]. Winer-Muram et al. reported a difference found of $20 \%$ in volume measurement of tumors between thin $(2-3 \mathrm{~mm})$ and thick (8-10) slice thickness settings [9]. Petrou et al. also reported similar findings [10], and Kuhnigk et al.[11] The NELSON trial, in which ten years follow up done came with a report that volume CT screening for indeterminate lung nodule growth rate assessment acts as an imaging biomarker that results in low referral along with lower lung-cancer mortality (in both sexes) than no screening, despite increased screening intervals over time.[12] Assessing the growth of nodules in volume is more approachable when nodules are spiculated or lobulated rather than round or spherical, where manual diameter measurement cannot predict the actual result [15].

This study found that pulmonary nodule whether benign or malignant has very different characteristics where solid and subsolid nodules are easier to diagnose in thin slice section thickness. For solid nodules, differential diagnosis is malignant (neoplastic) primary lung malignancies (non-small cell, small cell, carcinoid, lymphoma), solitary metastasis; benign causes like Hamartoma, AVM (arteriovenous malformation), infectious granuloma, round pneumonia, abscess; non-infectious Wegner's granulomatosis, infarct, rheumatoid nodule, amyloidosis; and congenital cause [26]. When the nodule shape is round or oval, it can be any of these; however, the nodule shape is an irregular probability of nodules, either primary lung cancer or inflammatory cause [27]. Nodule character can be further judged focusing on the internal feature as margin blurry or clear, presence of spiculation, lobulation [28]. If any nodule on CT is round or oval with a clear margin and no interior feature is seen, in that case, it is assumed to be benign or malignant due to any cause, and repeated follow-up is done to ensure that nodule is reducing in diameter or volume [29]. If the volume keeps increasing in repeated follow-up, it will be considered a malignant cause for primary lung cancer. Metastasis in the lung is usually blood-borne [30]. After reaching the lung capillaries, tumor cells infiltrate the vessel wall, grow in surrounding lung interstitial and alveoli, expand in size, compress surrounding structures, and form nodules.[13]. For persistent subsolid nodules, the differential cause may be malignant lung adenocarcinoma (pre-invasive lesions, AAH, AIS); metastasis from RCC, Melanoma, carcinoma of pancreas, breast, GIT. Benign causes can be organizing pneumonia, Focal interstitial fibrosis, endometriosis. Subsolid nodules containing components with ground-glass attenuation higher than that of lung parenchyma and lower than soft tissues may be pure GGN or have mixed solid nodules.[14] Most of the malignant tumors in this study are the subsolid type with irregular form. Nodules that were spiculated or lobulated most of them were malignant. Very few were benign with solid type were spiculated and lobulated. Benign nodules are mostly having air bronchogram where few nodules were attached with pleura and pleural retraction present. Meanwhile, malignant nodules having very few air bronchogram, but more were close to pleura and pleural retraction. However, these characteristics are very few but enough to determine and differentiate between benign and malignant [31].

Our study has some limitations. Benign nodules' sample size is minimal; therefore, more cases are needed for more accuracy. 


\section{Conclusion}

After the widespread use of multidetector computed tomography and growing interest in lung cancer screening of small pulmonary nodules is frequently detected. Recognition of early lung cancers is vital; size and growth are vital factors in assessing the malignant potential of a nodule. With increasing numbers of small pulmonary nodules being detected, clinicians will need more accurate prognostic prediction. An estimate of nodule diameter and volume is helpful when a clinician decides to use serial imaging to follow a screen-detected pulmonary nodule. To manage a pulmonary nodule giving more emphasis to volume change reduce unnecessary biopsy procedures in those with benign disease and promoting prompt management in malignancy.

The CT manifestations of different types of pulmonary nodules have certain characteristics. Understanding and grasping the signs of various types of nodules are of great value in the judgment and differential diagnosis of their properties to provide a more reliable reference basis for clinical treatment.

The volume measured by thin-slice CT images is a reliable indicator for evaluating the change in pulmonary nodules' follow-up. Accurately assessing the volume changes of pulmonary nodules combined with the CT manifestation will help improve pulmonary nodules' accuracy.

\section{Conflicts of Interest}

All the authors do not have any possible conflicts of interest.

\section{Guarantor}

Jannatul Maoya Bashanti had full access to all the data in the study and took responsibility for the data integrity and accuracy of the data analysis.

\section{Contributorship}

Jannatul Maoya Bashanti did the data collection and wrote the first draft of the manuscript. All authors reviewed and edited the manuscript and approved the final version of the manuscript.

\section{Acknowledgements}

We would like to thank Professor Lv Fajin for his assistance and guidance in this paper.

\section{References}

[1] Daiwei Han, Marjolein A Heuvelmans, Matthijs Oudkerk et al. Volume versus diameter assessment of small pulmonary nodules in C. T. lung cancer screening. Transl Lung Cancer Res. 2017; 6 (1): 52-61.
[2] Park EA, Goo JM, Lee JW, et al. Efficacy of computer-aided detection system and thin-slab maximum intensity projection technique in detecting pulmonary nodules in patients with resected metastases. Invest Radiol. 2009; 44 (3): 105-113.

[3] Tuddenham WJ et al. Glossary of terms for thoracic radiology: recommendations of the Nomenclature Committee of the Fleischner Society. AJR Am J Roentgenol. 1984; 143 (3): 509517.

[4] Hanamiya M, Aoki T, Yamashita Y, et al. Frequency and significance of pulmonary nodules on thin-section CT in patients with extrapulmonary malignant neoplasms. Eur J Radiol. 2012; 81 (1): 152-7.

[5] Aberle DR, Adams AM, Berg CD, et al. National Lung Screening Trial Research Team. Reduced lung-cancer mortality with low-dose computed tomographic screening. $\mathrm{N}$ Engl J Med. 2011; 365 (5): 395-409.

[6] Anna Rita Larici, Alessandra Farchione et al. Lung nodules: size still matters. Eur Respir Rev. 2017; 26 (1): 170025.

[7] Park CM, Goo JM, Lee HJ, et al. Nodular ground-glass opacity at thin-section CT: histologic correlation and evaluation of change at follow-up. Radiographics. 2007; 27 (2): 391-408.

[8] Hester A Gietema 1, Cornelia M Schaefer-Prokop, Willem P T M Mali, Gerard Groenewegen, Mathias Prokop et al. Pulmonary nodules: Interscan variability of semiautomated volume measurements with multisection CT- influence of inspiration level, nodule size, and segmentation performance. Radiol. 2007; 245 (3): 888-94.

[9] Winer-Muram HT, Jennings SG, Meyer CA, et al. effect of varying $\mathrm{CT}$ section width on volumetric measurement of lung tumors and application of compensatory equations. Radiology. 2003; 229: 184-94.

[10] Petrou M, Quint LE, Nan B, et al. Pulmonary nodule volumetric measurement variability as a function of $\mathrm{CT}$ slice thickness and nodule morphology. AJR Am J Roentgenol 2007; 188: 306-12.

[11] Kuhnigk JM, Dicken V, Bornemann L, et al. Morphological segmentation and partial volume analysis for volumetry of solid pulmonary lesions in thoracic CT scans. IEEE Trans Med Imaging 2006; 25: 417-34.

[12] Harry J de Koning, Carlijn M van der Aalst, Pim A de Jong, Ernst T Scholten, Kristiaan Nackaerts, Marjolein A Heuvelmans et al. Reduced Lung-Cancer Mortality with Volume CT Screening in a Randomized Trial. N Engl J Med. 2020 Feb; 382 (6): 503-513.

[13] Cai Minghui, Shen Tuyang et al. Clinical diagnosis and treatment strategies for metastatic lung tumors. Chinese Journal of Lung Cancer. 2014; 17 (3): 282-5.

[14] Mylene T. Truong, Jane P. Ko, Santiago E. Rossi, Ignacio Rossi, Chitra Viswanathan et al. Update in evaluating the solitary pulmonary nodule. RadioGraphics. Vol. 34, No. 6.

[15] Anand Devaraj, Bram van Ginneken, Arjun Nair et al. Use of volumetry for lung nodule management, Radiology rsna 2017, vol 284: No 3.

[16] Hiren j Mehta, James g Ravenel, Stephanie r shaftman et al. The utility of nodule volume in the context of malignancy prediction for small pulmonary nodules. CHEST 2014; 145 (3): 464-472. 
[17] James g Ravenel, William M leue, Paul J Nietert, James V Miller et al. Pulmonary nodule volume: effect of reconstruction parameters on the automated measurements-A phantom study. RSNA radiology volume 247; number 2- May 2008.

[18] Annemic Snoeckx, Peter reunites, Damien Desbuquoit, Maarten J Spinhoven et al. Evaluation of the solitary pulmonary nodule: size matters, but do not ignore the power of morphology. Insights imaging (2018) 9: 73-86.

[19] David F Yankelevitz, Rajiv Gupta, Binsheng Zhao, Claudia I Henschke et al. Small pulmonary nodules: Evaluation with repeat CT - preliminary experience. RSNA radiology 1999; 212: 561-566.

[20] Alexander A Bankier, Heber Macmohan, Jin Mo Goo, Geoffrey D Rubin. Recommendations for measuring pulmonary nodules at CT; a statement from the Fleischner society RSNA radiology volume 285 number 2- November 2017.

[21] Yoneda K, Ueno J, Nishihara S, et al. Post-processing technique with MDCT data improves the accuracy of detecting lung nodules. Radiat Med 2007; 25: 511-515.

[22] Verhagen MV, Smets AMJB, van Schuppen J, Deurloo EE, Schaefer-Prokop C. The impact of reconstruction techniques on observer performance for detecting and characterizing small pulmonary nodules in chest CT of children under 13 years. Eur J Radiol 2018; 100: 142-146.

[23] Angelelli G, Grimaldi V, Spinelli F, Scardapane A, Sardar A. Multislice computed tomography in the study of pulmonary metastases. Radiol Med (Torino) 2008; 113: 954-967.
[24] Chang B, Hwang JH, Choi YH, et al. Natural history of pure ground-glass opacity lung nodules detected by low-dose CT scan. Chest 2013; 143: 172-178.

[25] Goo JM. A computer-aided diagnosis for evaluating lung nodules on chest CT: the current status and perspective. Korean J Radiol 2011; 12: 145-155.

[26] Tammemagi MC, Katki HA, Hocking WG, et al. Selection criteria for lung-cancer screening [J]. N Engl J Med, 2013, 368 (8): 728-736.

[27] Zhi-gang chu; yan zhang; Wang-Jia li; Qi Li et al. Primary solid lung cancerous nodules with different sizes. BMC 2019, 191060 .

[28] Wang-Jia Li, Zhi-gang Chu, Qi Li, and Fa-jin Lv et al. Effect of Slab Thickness on the Detection of Pulmonary Nodules using CT Maximum and Minimum Intensity Projection. American Journal of Roentgenology. 2019; 213: 562-567.

[29] Jing Yang, Hailin wang, Chen Geng, Yakang Dai et al. Advances in intelligent diagnosis methods for pulmonary ground-glass nodules. Biomed eng online 2018 17; 20.

[30] Mingzhu Liang, Rowena Yi, Wei tang et al. Variation in screening CT-detected nodule volumetry as a function of size; AJR 2017; 209: 304-308.

[31] Takashi Eguchi, Akihiko Yoshizawa, Satoshi Kawakami et al. Tumor size and computed tomography attenuation of pure pulmonary GGN are useful for predicting pathological invasiveness. PLoS One. 2014 May 20; 9 (5): e97867. 